\title{
Characteristics of intestinal Behçet's Disease and the comparison of different medications
}

\author{
Hanjiang Zeng ${ }^{1}$, Jun Zhou ${ }^{1}$, Yu Wang ${ }^{2}$, Yiming Liu' ${ }^{2}$, Fan Lian ${ }^{1 *}$ \\ ${ }^{1}$ Department of Rheumatology \& Clinical Immunology, the First Affiliated Hospital of Sun Yat-sen University, Guangzhou, China \\ ${ }^{2}$ Department of Interventional Oncology, the First Affiliated Hospital of Sun Yat-sen University, Guangzhou, China
}

\begin{abstract}
Objective: To evaluate the clinical characteristics of gastrointestinal Behcet's disease in a cohort of South China and compare the efficacy of different medications.

Method: We retrospectively revised the medical record of a cohort of BD patients admitted to the first affiliated university hospital of Sun Yat-sen University between January 2005 and March 2017. Demographic information, gastrointestinal symptoms and extra-intestinal manifestations, laboratory findings, disease activity index (DAIBD), imaging characteristics and managements were documented.

Results: Records of 1648 Behcet's disease cases were reviewed, of which, 187 cases were diagnosed of intestinal BD. Intestinal BD was more commonly found in male (73.3\%) and male showed a significantly higher rate of gastrointestinal haemorrhage. Higher frequency of eye involvements and musculoskeletal involvements, less skin lesions were noted in the intestinal BD group. Abdominal pain and abdominal distension were the most common symptoms observed. Ileal/Ileocecal region was the most commonly affected under endoscopy. Mesenteric vessel lesions were not frequently detected but associated with poor prognosis. Most intestinal BD cases could be controlled with corticosteroid and immunosuppressors. CTX showed longer recurrence duration than AZA or MTX as maintenance therapy.

Conclusion: Intestinal Behcet's diseases could be difficult to diagnosis and associated with severe complications. Male is more commonly involved and had higher rate of severe complications. Mesenteric vessel lesions were not frequently detected but associated with poor prognosis. Corticosteroids, immunosuppressants and TNF blockers were effective for most patients. As maintenance therapy, CTX was more effective than AZA or MTX.
\end{abstract}

\section{Introduction}

Behçet's disease (BD) is an inflammatory disorder of unknown etiology classically characterized by recurrent oral, genital ulcers, uveitis and skin lesions. Behçet's patients can present with arthritis, gastrointestinal, neurological and vascular involvements as well [13]. There is no specific laboratory or pathological laboratory tests for Behcet's disease. Diagnosis is based on the observation of clinical manifestation and/or pathergy test. Intestinal $\mathrm{BD}$ is a subtype of $\mathrm{BD}$ characterized by gastrointestinal involvements, with an incidence of approximately 3-16\% [1]. Gastrointestinal (GI) manifestations of Behçet's disease are of particular importance as they could be associated with serious complications. The clinical manifestation of gastrointestinal involvements varies greatly, from mild symptoms to perforation, infarction and massive bleeding [4]. Intestinal BD is not easy to reach prompt and correct diagnosis when gastroenteropathy is presented as the initial or predominant manifestation. Intestinal ulcers may be resistant to medical therapy and with high rate of recurrence [5]. Timely and adequate treatment can alleviate the clinical symptoms and improve the prognosis.

However, optimal medical treatment for intestinal BD has not been extensively identified and established. Systemic corticosteroids and immunosuppressant are the main empiric therapeutic agents [6]. Anti-TNF- $\alpha$ mAb may have therapeutic potential for inducing and maintaining remission $[7,8]$.

Surgery is an option for fulminant cases, though a higher risk of recurrence is documented $[9,10]$. Treatments with long-term effectiveness and safety are required to be introduced for better therapeutic outcomes in intestinal BD.

The data on the clinical features, outcomes and therapy of $\mathrm{BD}$ patients with gastrointestinal involvements in Chinese population are not yet sufficient. The present study retrospectively reviewed intestinal BD patients in a Chinese population, evaluate the clinical characteristics and putative associated factors contributing to early identification, then compare the efficacy and safety profiles of different treatment strategies, and thus, hopefully allow for the improvement of prompt diagnosis and favourable outcomes.

\section{Methods}

\section{Patients}

A retrospective cohort study was conducted. Records of 1648 Behcet's disease cases consecutively admitted to the first affiliated hospital of Sun Yat-sen University, Guangzhou, China, between January 2005 and March 2017 were reviewed. The study protocol

*Correspondence to: Fan Lian, Department of Rheumatology \& Clinical Immunology, the First Affiliated Hospital of Sun Yat-sen University, Guangzhou, China, Tel: 008613922263595; E-mail: lianfan_1@hotmail.com

Key words: intestinal Behcet's disease, clinical characteristics, endoscopy findings, recurrence, maintenance therapy

Received: December 21, 2018; Accepted: December 28, 2018; Published: December 31, 2018 
was approved by the Ethics Committee of the first affiliated hospital of Sun Yat-sen University. BD was defined by the International Study Group criteria for BD (1990) [11]. Demographic data, gastrointestinal symptoms and extra-intestinal manifestations were recorded. Diagnosis of intestinal $\mathrm{BD}$ was made according to the criteria as described using a modified Delphi process [12,13]. Intestinal BD was defined as mucosal inflammation and ulcer formation on endoscopy or mesenteric artery involvements identified on abdominal computed tomography (CT) [13-15]. Patients with a history of previous colon operation, diagnosed or suspected UC, CD, intestinal tuberculosis, infectious enteritis, and malignancy were excluded.

DAIBD was used to evaluate clinical activity of intestinal BD. The following eight variables were documented: general well-being, fever, extraintestinal manifestations, abdominal pain, abdominal mass, intestinal complications, and number of liquid stools in one week. The DAIBD was classified into 3 levels: quiescent

\section{( $\leq 19)$, mild (20-39), moderate (40-74), and severe ( $\geq 75)$ [16].}

Clinical remission was defined as the GI symptom remission and normal serum CRP level. Clinical response was defined as the alleviation of GI symptoms and lower CRP level. Clinical relapse was defined as the exacerbation of GI symptoms plus serum CRP elevation or relapse of GI lesions confirmed by imaging studies that lead to more aggressive treatment. The cumulative probability of recurrence was calculated based on the interval between the clinical remission and the time of GI symptom recurrence [17].

\section{Endoscopy}

Endoscopic examination was performed in all intestinal BD subjects. Localized type of ulceration was defined when lesions were restricted to only one segment and a diffuse type was defined when lesions were scattered in more than two segments [18], when two or more ulcers were observed, the largest diameter of the biggest ulcer was used for the measurement of depth, shape, size and margin for analysis [19].

\section{Statistical analysis}

Continuous variables are presented as mean \pm SD and categorical variables are presented as frequency and percentage. Numerical analysis was performed using the Mann-Whitney test or Student's $\mathrm{t}$-test. Categorical analysis was performed using the the $\chi^{2}$ or Fisher's exact test. The cumulative probabilities of clinical relapse, recurrence was calculated using the Kaplan-Meier method. Statistical significance was set at $\mathrm{P}<0.05$. All calculations were performed using SPSS Version 23.0 (SPSS, IBM Corporation, New York).

\section{Results}

\section{Demographic and clinical characteristics of intestinal Behçet's Disease}

Altogether 1648 cases of Behcet's disease cases were reviewed, in which $187(187 / 1648,11.3 \%)$ cases were diagnosed of intestinal BD. Intestinal BD were more commonly found in male (137/187, 73.3\%) than in female (50/187, 26.7\%). Male/female ratio was similar and held no statistical significance in the intestinal $\mathrm{BD}$ group and $\mathrm{BD}$ patients without gastrointestinal involvements. The mean age at the onset of GI involvements was $31.26 \pm 15.72$ years. The median time between the diagnosis of $\mathrm{BD}$ and GI involvements was 2 months, ranged from 0 to 186 months. The most and second frequent manifestations in the two groups were recurrent oral ulcers and genital ulcers (no significant difference). Higher frequency of eye involvements and musculoskeletal involvements were noted in the intestinal $\mathrm{BD}$ group compared to the control group $(\mathrm{p}<0.05)$. Much less skin lesions were observed in intestinal BD group $(\mathrm{p}<0.05)$. Vascular disorders such as thrombosis and aneurysm were slightly more in intestinal $\mathrm{BD}$ group than in the control group (not significant, $\mathrm{p}>0.05$ ), though patients with vascular involvements in other parts of the body were more inclined to complicate with mesenteric lesions. There were no significant differences between the two groups in genitourinary involvements and CNS involvements. Table 1 compared the demographic and clinical characteristics of intestinal BD with BD without GI involvements.

Among the 187 cases of intestinal BD, all of them underwent endoscopy and CT scan to confirm the diagnosis. Gastrointestinal manifestations were summarized in Table 2. Abdominal pain and abdominal distension were the most common symptoms observed. Gastrointestinal haemorrhage happened in more than $30 \%$ of the patients. Male showed a significantly higher rate of gastrointestinal haemorrhage ( 49 cases of male GI bleeding versus 13 cases of female GI bleeding).

Endoscopy findings (Table 3) shown that the Ileal/Ileocecal region was the most commonly affected. Other parts of small intestine were frequently involved. Most lesions were large $(>1 \mathrm{~cm})$, deep, localized, round or oval shape.

Oesophagus and stomach were rarely involved. No rectal lesion was found. Retrosternal chest pain was associated with oesophageal

Table 1. Demographic and clinical characteristics of intestinal Behcet's disease

\begin{tabular}{|c|c|c|c|}
\hline Demographic and clinical characteristics & Gastrointestinal BD $(n=187)$ & $\begin{array}{l}\text { BD without gastrointestinal } \\
\text { involvements }(n=1461)\end{array}$ & P-value \\
\hline Male, $n(\%)$ & $137(73.3)$ & $998(68.3)$ & $>0.05$ \\
\hline $\mathrm{CRP}(\mathrm{mg} / \mathrm{L})$, mean $\pm \mathrm{SD}$ & $42.37 \pm 37.35$ & $38.53 \pm 32.72$ & $>0.05$ \\
\hline $\operatorname{ESR}(\mathrm{mm} / \mathrm{h})$, mean $\pm \mathrm{SD}$ & $52.37 \pm 37.93$ & $46.31 \pm 27.77$ & $>0.05$ \\
\hline Recurrent oral ulcer, $\mathrm{n}(\%)$ & $183(96.8)$ & $1425(97.5)$ & $>0.05$ \\
\hline skin lesion, $\mathrm{n}\left({ }^{\%}\right)^{*}$ & $36(19.3)$ & $381(26.1)$ & $<0.05$ \\
\hline Ocular lesion, $\mathrm{n}(\%)^{*}$ & $42(22.5)$ & $217(14.8)$ & $<0.05$ \\
\hline Musculoskeletal involvements, $\mathrm{n}(\%)^{*}$ & $102(54.5)$ & $306(20.9)$ & $<0.05$ \\
\hline Genitourinary involvements, $\mathrm{n}(\%)$ & $115(61.5)$ & $982(67.2)$ & $>0.05$ \\
\hline Cardiac involvement, $\mathrm{n}(\%)$ & $0(0)$ & $2(0.1 \%)$ & $>0.05$ \\
\hline Lung involvement, $\mathrm{n}(\%)$ & $0(0)$ & $0(0)$ & $>0.05$ \\
\hline Central nervous system involvements, $\mathrm{n}(\%)$ & $19(10.2)$ & $137(9.4)$ & $>0.05$ \\
\hline Vascular disorders (thrombosis, aneurysm, dissection, etc), n (\%) & $111(59.3)$ & $745(51.0)$ & $>0.05$ \\
\hline Disease activity (DAIBD) at the time of diagnosis, mean \pm SD & $71.6 \pm 43.4$ & & \\
\hline
\end{tabular}


Table 2. Gastrointestinal manifestations of intestinal Behcet's disease

\begin{tabular}{|l|l|}
\hline Symptoms & Number (\%) \\
\hline Abdominal pain & $135(72.2)$ \\
\hline Abdominal distension & $67(35.8)$ \\
\hline Dyspepsia & $44(23.5)$ \\
\hline Melena/hematochezia & $62(33.2)$ \\
\hline Dysphagia & $7(3.7)$ \\
\hline Nausea/vomiting & $21(11.2)$ \\
\hline Hematemesis & $3(1.6)$ \\
\hline Abdominal mass & $17(9.1)$ \\
\hline Diarrhea & $65(34.8)$ \\
\hline Retrosternal chest pain & $5(2.6)$ \\
\hline
\end{tabular}

Table 3. Endoscopy findings of intestinal Behcet's disease

\begin{tabular}{|l|l|}
\hline Endoscopy findings & Number (\%) \\
\hline Esophageal ulcerations & $9(4.8)$ \\
\hline Gastroduodenal ulcerations & $7(3.7)$ \\
\hline Jejunum ulcerations & $93(49.7)$ \\
\hline Ileal/Ileocecal ulcerations & $157(83.9)$ \\
\hline Colonic ulcerations & $29(15.5)$ \\
\hline Rectal ulcerations & $0(0.0)$ \\
\hline Number of ulcers & \\
\hline Solitary & $109(58.3)$ \\
\hline $02-05$ & $46(24.6)$ \\
\hline$\geq 5$ & $32(17.1)$ \\
\hline Localized involvements & $121(64.7)$ \\
\hline Diffuse involvements & $66(35.3)$ \\
\hline Deep ulcer & $117(62.6)$ \\
\hline Superficial ulcer & $70(37.4)$ \\
\hline Ulcers $>1$ cm & $145(77.5)$ \\
\hline Abscess formation & $1(0.5)$ \\
\hline Perforation & $2(1.0)$ \\
\hline Stricture & $2(1.0)$ \\
\hline & \\
\hline
\end{tabular}

ulcerations. Oesophageal ulcers tend to localize in the middle or lower part of the oesophagus. Severe complications such as bowel stricture, fistula, perforations or abscess formation were rare.

CT scanning was summarized in Table 4. Mesenteric vessel lesions were not frequently seen. However, of all the 26 cases with abnormal vessel findings on CT scan, remission rates 8 weeks were less than $30 \%$ (7/26, 26.9\%). Twenty-three cases had multiple relapses, 3 cases exhibited intestinal infarction, and one died.

\section{Treatment and outcome}

Most intestinal $\mathrm{BD}$ cases could be controlled if appropriate treatment was applied. Corticosteroids and immunosuppressants were given based on the clinical course. No recurrence was observed in 103 patients during the follow up. Altogether 4 patients went through inevitable death. Side effects (severe infection, 2 cases) and irreversible intestinal damage ( 2 cases) were the main causes. Among the 65 cases of GI bleeding, 59 cases were suppressed with corticosteroids and immunosuppressants including cyclophosphamide, methotrexate, or azathioprine. Two cases failed the traditional therapy yet could be controlled by TNF-a blocker (one with infliximab and one with adalimumab) plus immunosuppresant. Four cases of GI bleeding underwent surgery eventually, and one died. These four cases were all young ( $<30$ years old), male, with high CRP $(>100 \mathrm{mg} / \mathrm{mL})$, and lack of initial response to traditional therapy. Severe complication included one abscess formation, 2 strictures and 2 perforations. Surgery was performed, and one patient of perforation didn't survive.
Azathioprine (AZA), cyclophosphamide (CTX) and methotrexate (MTX) were used for maintenance therapy. The median recurrence duration among the three groups were presented in Figure 1.

Among the 84 patients underwent recurrence, those treated with CTX showed longer duration than AZA or MTX. The median recurrence duration for the medications above was: 14 months for CTX vs 10 months for AZA and 8 months for MTX 8 months, respectively.

We found significant difference between CTX group and the other two groups (CTX vs AZA p=0.049; CTX vs MTX $p=0.014$ ), but the comparison between AZA group and MTX group did not reach a statistical significance (AZA vs MTX p=0.507).

\section{Discussion}

Gastrointestinal Behcet disease is not rare but could be difficult to diagnosis. Intestinal BD could possibly lead to fatal outcomes, and the prognosis varies. The prevalence of BD was reported $14 / 100000$ in China [20]. The frequency of intestinal BD varies from $1-60 \%$ in different countries [5]. A moderate frequency around 10\% was reported from China, which was consistent with our data calculated as $11.3 \%$ [21]. Male is dominant $(137,73.3 \%)$ in our study, yet no significant difference was found when compared to the BD population without gastrointestinal involvement. However, severe complication such as massive bleeding, abscess formation, and perforation happened much more frequently in male than in female. Patients couldn't sustain clinical remission were mostly male. Our data suggested that male intestinal BD patients may experience a relatively poor prognosis and requires more intensive monitoring compared to female.

Higher frequency of eye involvements and musculoskeletal involvements were noted in the intestinal BD group. It's reasonable to be alert of the gastrointestinal lesions if $\mathrm{BD}$ patients complain of the manifestations mentioned above.

Intestinal $\mathrm{BD}$ can be a difficult diagnosis to establish sometimes, with unspecific manifestations such as abdominal pain, abdominal distension and dyspepsia, etc., which were the dominant symptoms

Table 4. CT scanning of intestinal Behcet's disease

\begin{tabular}{|l|l|}
\hline CT scanning & Number (\%) \\
\hline Mesenteric artery aneurysm & $7(3.7)$ \\
\hline Mesenteric artery dissection & $5(2.7)$ \\
\hline Mesenteric thrombosis & $14(7.5)$ \\
\hline
\end{tabular}
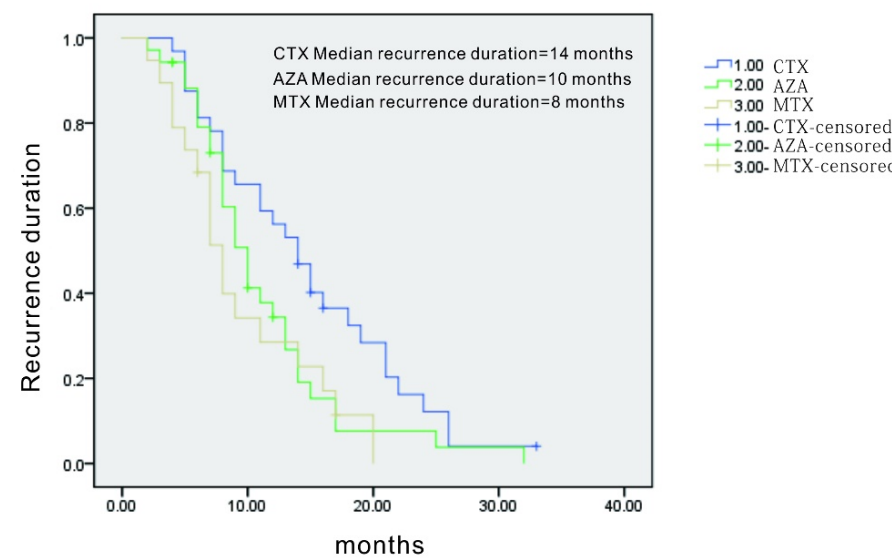

Figure 1. Kaplan analysis for the comparison of three immunosuppressants in maintenance therapy. CTX group had the longest recurrence duration compared to the other two AZA and MTX 
documented we observed, also these are the symptoms could happen in patients suffered from side effects of medications, or Crohn's disease, the main differential diagnosis for intestinal BD [5].

Behcet's disease is a vasculitis which can affect vessels of all types and sizes including mesenteric vessels. Siğirci A, et al. suggested that $\mathrm{BD}$ patients who had gastrointestinal symptoms were associated with change of mesenteric blood flow in Doppler examination [22]. We observed several mesenteric lesions on CT scanning, including aneurysm, dissection and thrombosis. Mesenteric artery disease can result in intestinal ischemia and infarction [23]. Abdominal vessel lesions were associated with more severe clinical course, higher surgical rate and more relapse, and there may be a need for more radical therapeutic agents.

Endoscopy and CT scanning is useful for therapeutic decisions. Although routine endoscopy is not recommended for BD, endoscopy and abdominal CT for patients with gastrointestinal symptoms may be appropriate $[24,25]$. And colonoscopy can help to differentiate CD and intestinal BD [26]. Previous research suggested that any part of the intestine may be affected, mainly in terminal ileum and cecum. Gastrointestinal involvements could emerge at various time points throughout the disease course [21]. We observed and confirmed the previous studies. Ileal/ileocecal region was the most commonly affected. Other parts of small intestine were frequently involved. Oesophagus and stomach lesions were rarely seen. No rectal lesion was found. Ileocecal area ulcers $(<5)$ that were oval in shape, deep, and with discrete borders were considered typical intestinal BD lesions [27]. Some other unspecific ulceration we observed could be multiple, shallow, small or large, and unclearly marginated. When systemic manifestations, disease duration, or lesions of other organs were examined, such involvements may not be correlated with gastrointestinal flares.

High disease activity (DAIBD $\geq 40)$ were reported to be associated with intestinal $\mathrm{BD}$, a more severe clinical course, lower complete remission rate and inevitable surgery $[9,18,28-30]$. Our results showed that DAIBD was high in intestinal BD, but CRP/ESR exhibited no significance when compared to $\mathrm{BD}$ without gastrointestinal involvements, implying that high disease activity didn't necessarily indicate the occurrence of intestinal BD.

GI bleeding was common in the present study. Over $90 \%$ of bleedings were well managed with traditional medications corticosteroids and immunosuppressant. Serious complications necessitate unavoidable operative management such as stricture, abscess formation, or perforations were not commonly seen in our research, far less than previous studies have reported the rates of perforation, fistula, stricture and abscess to be $12.7 \%, 7.6 \%, 7.2 \%$ and $3.3 \%$ respectively [31].

Previous studies have shown that the cumulative rates of surgical intervention are $20 \%$ at 1 year, $27-33 \%$ at 5 years, and $31-46 \% 10$ years after the diagnosis of intestinal BD [32]. Our results provided dissimilar evidence that less than $10 \%$ of the intestinal BD underwent surgery eventually. And it was hard to indicate the predictive factor for surgery with such a small number of patients involved in operational management.

Intestinal $\mathrm{BD}$ exhibits a chronic and recurrent disease course, with considerable variability of prognosis. Prompt diagnosis and appropriate tailored treatment would help with stable remission and prevention of irreversible intestinal damage. Management of intestinal BD is not yet elucidated because there is no internationally accepted, standardized treatment strategies [33]. Anti-TNF- $\alpha$ mAb therapy alone or in combination with immunosuppressant was reported and replicated [34-40]. Two cases in our research failed the traditional therapy yet could be controlled by TNF- $\alpha$ blocker (one with infliximab and one with adalimumab). However, the treatment response is controversial, and four cases failed all the medical treatment thus surgery was indicated. Younger age, male and very high CRP may be poor prognostic factor, but the case load is too small to explicate the predictive power. Still, these may be indicators of early use of immunosuppressant and intensive patient monitoring in daily medical practice.

There is still a lack of uniform consensus on maintenance therapy of intestinal BD. It was suggested that the same classes of medications that have been used for the treatment of systemic BD could also been used to treat intestinal BD [21].

The current study indicated that more than $50 \%$ of the patients sustained remission when treated with regular immunosuppressant, and CTX used as maintenance therapy was associated with longer recurrence duration than AZA or MTX. Most of the recurrent patients had the symptoms back in one and a half year, implying this period was critical to disease monitoring. Longer follow-up periods and bigger population may be required to see significant outcomes.

\section{Conclusion}

In conclusion, gastrointestinal Behcet's diseases could be difficult to diagnosis and associated with severe complications. Male is dominant in our study. Severe complication and recurrence happened much more frequently in male than in female. Higher frequency of eye involvements and musculoskeletal involvements were noted in the intestinal BD group. Mucosal inflammation/ulcers of intestinal tract and mesenteric vessel disease were two forms of gastrointestinal Behcet's disease. Any part of the intestine could be affected. Ileal/ileocecal lesion was the most commonly seen. Mesenteric vessel lesions were not frequently detected, including aneurysm, dissection and thrombosis, but they were associated with poor prognosis. GI bleeding was common in the present study. Corticosteroids, immunosuppressants and TNF blockers were effective for most patients. As maintenance therapy, CTX was associated with longer recurrence duration than AZA or MTX.

\section{Acknowledgements}

Not applicable.

\section{Funding}

Project supported by National Natural Science Foundation of China (No. 81102270) and Guangdong Natural Science Foundation (No. 2016A030313217).

\section{Availability of data and materials}

The datasets used and/or analyzed during the current study are available from the corresponding author on reasonable request, but no information infringing on the privacy of the participants will be given.

\section{Consent for publication}

All authors agreed to submit in present form and signed to the copyright of "Rheumatology and Orthopedic Medicine". The authors all consented to publish.

\section{References}

1. Sakane T, Takeno M, Suzuki N, Inaba G (1999) Behçet's disease. N Engl J Med 341: 1284-1291. [Crossref] 
2. Saleh Z, Arayssi T (2014) Update on the therapy of Behçet disease. Ther Adv Chronic Dis 5: 112-134. [Crossref]

3. Hamdan A, Mansour W, Uthman I, Masri AF, Nasr F, et al. (2006) Behçet's disease in Lebanon: clinical profile, severity and two-decade comparison. Clin Rheumatol 25: 364-367. [Crossref]

4. Wu QJ, Zhang FC, Zhang X (2012) Adamantiades-Behcet's disease-complicated gastroenteropathy. World J Gastroenterol 18: 609-615.

5. Bayraktar Y, Ozaslan E, Van Thiel DH (2000) Gastrointestinal manifestations of Behcet's disease. J Clin Gastroenterol 30: 144-154. [Crossref]

6. Okada AA (2006) Behçet's disease: general concepts and recent advances. Curr Opin Ophthalmol 17: 551-556.

7. Yamashita N, Kaneoka H, Kaneko S, Takeno M, Oneda K, et al. (1997) Role of gammadelta T lymphocytes in the development of Behçet's disease. Clin Exp Immunol 107: 241-247. [Crossref]

8. Travis SP, Czajkowski M, McGovern DP, Watson RGP, Bell AL (2001) Treatment of intestinal Behcet's syndrome with chimeric tumour necrosis factor alpha antibody. Gut 49: 725-728.

9. Jung YS, Yoon JY, Lee JH, Jeon SM, Hong SP, et al. (2011) Prognostic factors and long-term clinical outcomes for surgical patients with intestinal Behcet's disease. Inflamm Bowel Dis 17: 1594-1602.

10. Naganuma M, Iwao Y, Inoue N, Hisamatsu T, Imaeda H, et al. (2000) Analysis of clinical course and long-term prognosis of surgical and nonsurgical patients with intestinal Behçet's disease. Am J Gastroenterol 95: 2848-2851.

11. International Study Group for Behçet's Disease (1990) Criteria for diagnosis of Behçet's disease. Lancet 335: 1078-1080. [Crossref]

12. Kobayashi K, Ueno F, Bito S, Iwao Y, Fukushima T, et al. (2007) Development of consensus statements for the diagnosis and management of intestinal Behçet's disease using a modified Delphi approach. $J$ Gastroenterol 42: 737-745.

13. Cheon JH, Kim ES, Shin SJ, Kim TI, Lee KM, et al. (2009) Development and validation of novel diagnostic criteria for intestinal Behçet's disease in Korean patients with ileocolonic ulcers. Am J Gastroenterol 104: 2492-2499.

14. Vaiopoulos AG, Sfikakis PP, Kanakis MA, Vaiopoulos G, Kaklamanis PG (2014) Gastrointestinal manifestations of Behçet's disease: advances in evaluation and management. Clin Exp Rheumatol 32: S140-S148.

15. Hisamatsu T, Naganuma M, Matsuoka K, Kanai T (2014) Diagnosis and management of intestinal Behçet's disease. Clin J Gastroenterol 7: 205-212. [Crossref]

16. Cheon JH, Han DS, Park JY, Ye DB, Jung SA, et al. (2011) Development, validation, and responsiveness of a novel disease activity index for intestinal Behçet's disease. Inflamm Bowel Dis 17: 605-613.

17. Kinoshita H, Kunisaki R, Yamamoto H, Matsuda R, Sasaki, et al. (2013) Efficacy of infliximab in patients with intestinal Behçet's disease refractory to conventional medication. Intern Med 52: 1855-1862.

18. Kim JS, Lim SH, Choi IJ, Moon H, Jung CH, et al. (2000) Prediction of the clinical course of Behçet's colitis according to macroscopic classification by colonoscopy. Endoscopy 32: 635-640.

19. Lee HJ, Kim YN, Jang HW, Jeon HH, Jung ES, et al. (2012) Correlations between endoscopic and clinical disease activity indices in intestinal Behcet's disease. World $J$ Gastroenterol 18: 5771-5778.

20. Zhang Z, He F, Shi Y (2013) Behcet's disease seen in China: analysis of 334 cases. Rheumatol Int 33: 645-648. [Crossref]

21. Skef W, Hamilton MJ, Arayssi T (2015) Gastrointestinal Behçet's disease: a review. World J Gastroenterol 21: 3801-3812. [Crossref]
22. Sigirci A, Senol M, Aydin E, Kutlu R, Alkan A, et al. (2003) Doppler waveforms and blood flow parameters of the superior and inferior mesenteric arteries in patients having Behçet disease with and without gastrointestinal symptoms: preliminary data. $J$ Ultrasound Med 22: 449-457.

23. Vaiopoulos AG, Sfikakis PP, Kanakis MA, Vaiopoulos G, Kaklamanis PG (2014) Gastrointestinal manifestations of Behçet's disease: advances in evaluation and management. Clin Exp Rheumatol 32: S140-S148.

24. Houman MH, Ben Ghorbel I, Lamloum M, Khanfir M, Braham A, et al. (2002) Esophageal involvement in Behcet's disease. Yonsei Med J 43: 457-460. [Crossref]

25. Bottomley WW, Dakkak M, Walton S, Bennett JR (1992) Esophageal involvement in Behçet's disease. Is endoscopy necessary? Dig Dis Sci 37: 594-597. [Crossref]

26. Lee SK, Kim BK, Kim TI, Kim WH (2009) Differential diagnosis of intestinal Behçet's disease and Crohn's disease by colonoscopic findings. Endoscopy 41: 9-16.

27. Lee CR, Kim WH, Cho YS, Kim MH, Kim JH, et al. (2001) Colonoscopic findings in intestinal Behçet's disease. Inflamm Bowel Dis 7: 243-249. [Crossref]

28. Jung YS, Cheon JH, Park SJ, Hong SP, Kim TI, et al. (2013) Clinical course of intestina Behcet's disease during the first five years. Dig Dis Sci 58: 496-503. [Crossref]

29. Moon CM, Cheon JH, Shin JK, Jeon SM, Bok HJ, et al. (2010) Prediction of free bowel perforation in patients with intestinal Behçet's disease using clinical and colonoscopic findings. Dig Dis Sci 55: 2904-2911. [Crossref]

30. Park JJ, Kim WH, Cheon JH (2013) Outcome predictors for intestinal Behçet’s disease. Yonsei Med J 54: 1084-1090. [Crossref]

31. Jung YS, Cheon JH, Park SJ, Hong SP, Kim TI, et al. (2013) Long-term clinical outcomes of Crohn's disease and intestinal Behcet's disease. Inflamm Bowel Dis 19: 99-105. [Crossref]

32. Jung YS, Yoon JY, Hong SP, Kim TI, Kim WH, et al. (2012) Influence of age at diagnosis and sex on clinical course and long-term prognosis of intestinal Behçet's disease. Inflamm Bowel Dis 18: 1064-1071.

33. Hatemi G, Silman A, Bang D, et al. (2009) Management of Behçet disease: a systematic literature review for the European League Against Rheumatism evidence-based recommendations for the management of Behçet disease. Ann Rheum Dis 68: 15281534.

34. Iwata S, Saito K, Yamaoka K, Tsujimura S, Nawata M, et al. (2009) Effects of antiTNF-alpha antibody infliximab in refractory entero-Behcet's disease. Rheumatology 48: 1012-1013.

35. Kinoshita H, Kunisaki R, Yamamoto H, Matsuda R, Sasaki T, et al. (2013) Efficacy of infliximab in patients with intestinal Behçet's disease refractory to conventional medication. Intern Med 52: 1855-1862.

36. Lee JH, Cheon JH, Jeon SW, Ye BD, Yang S-K, et al. (2013) Efficacy of infliximab in intestinal Behçet' s disease: a Korean multicenter retrospective study. Inflamm Bowel Dis 19: 1833-1838.

37. De Cassan C, De Vroey B, Dussault C, Hachulla E, Buche S, et al. (2011) Successful treatment with adalimumab in a familial case of gastrointestinal Behcet's disease. $J$ Crohns Colitis 5: 364-368.

38. Ariyachaipanich A, Berkelhammer C, Nicola H (2009) Intestinal Behçet's disease: maintenance of remission with adalimumab monotherapy. Inflamm Bowel Dis 15: $1769-1771$

39. van Laar JA, Missotten T, van Daele PL, Jamnitski A, Baarsma GS, et al. (2007) Adalimumab: a new modality for Behçet's disease? Ann Rheum Dis 66: 565-566. [Crossref]

40. Shimizu Y, Takeda T, Matsumoto R, Yoshida K, Nakajima J, et al. (2012) Clinical efficacy of adalimumab for a postoperative marginal ulcer in gastrointestinal. Behçet disease. Nihon Shokakibyo Gakkai Zasshi 109: 774-780.

Copyright: (C2018 Zeng H. This is an open-access article distributed under the terms of the Creative Commons Attribution License, which permits unrestricted use, distribution, and reproduction in any medium, provided the original author and source are credited. 